\title{
A Flux Preserving Method of Coupling First and Second Order Equations to Simulate the Flow of Plasma between the Protonosphere and the lonosphere
}

\author{
E. R. YOUNG*, P. G. RichaRds, AND D. G. TORR ${ }^{+}$ \\ Space Physics Research Laboratory, Department of Atmospheric \\ and Oceanic Science, University of Michigan, Ann Arbor, Michigan 48109
}

Received May 15, 1979; revised December 3, 1979

\begin{abstract}
This paper describes a numerical method developed to solve the interhemispheric flow of thermal plasma, heat and momentum along closed magnetic field tubes in the plasmasphere. The essence of our technique incorporates the best aspects of two former approaches into a single unified code. The first is the so-called shooting or searching method, which employs integro-differential equations, and the second involves the solution of second order nonlinear partial differential equations by conventional iterative techniques. The former method attains optimal performance above $\sim 2000 \mathrm{~km}$, and the latter below this altitude. The combined approach yields a satisfactory solution over an entire geomagnetic flux tube, encompassing two low altitude regimes, in the northern and southern ionospheres, and a high altitude regime spanning the distance between them. We demonstrate that the solution is stable and simulate the collapse of the postsunset ionosphere.
\end{abstract}

\section{INTRODUCTION}

In this paper we present the numerical solution of a system of equations which describes the coupling of heat flow, momentum transfer and plasma densities between the ionosphere and magnetosphere. Despite significant advances over the last decade, which have been described in a number of recent reviews $[1-4]$, the coupling mechanisms have not been studied using a realistic formulation which simultaneously acounts for variations in all the major controlling variables.

A major shortcoming in previous studies has been the use of ad hoc boundary conditions, which generally arise when only part of a field line is modeled. We surmount this limitation in our treatment by simulating the flow and density in an entire flux tube spanning the midlatitude plasmasphere between magnetically conjugate points in the $\mathrm{F}$ regions of the northern and southern ionospheres, as shown in Fig. 1. The solution encompasses several regions of differing dominant physical

* Permanent address: Regis College Research Center, Weston, Mass.

"Also affiliated with: National Institute for Telecommunications Research of the CSIR, Johannesburg, South Africa. 


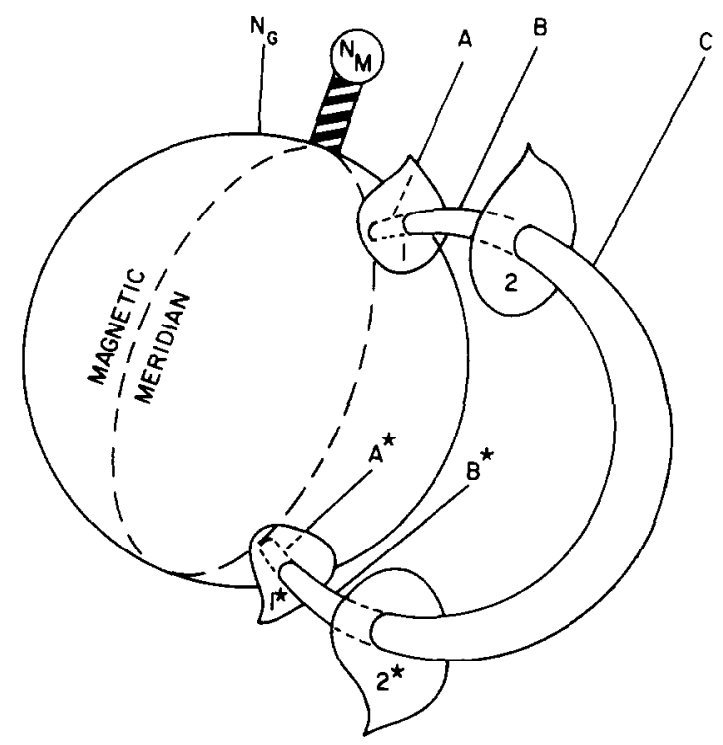

FIG. 1. Regions and boundaries. A, A*: Regions of local chemistry. B, B*: Ion diffusion regions where parabolic density equations can be formulated. C: Dynamic equilibrium region. $1,1^{*}, 2,2^{*}$ : Boundaries between adjacent regions at 1 and $1 *$. Densities are continuous while at 2 and $2^{*}$ both density and flux are continuous.

processes, which in turn require different formulations and numerical treatment. We use an optimal mathematical approach for each altitude regime, and derive the appropriate and unique boundary conditions which link these regions.

In the section immediately following we present the formulation of the plasma equations which describe the densities and flow of $\mathrm{O}^{+}, \mathrm{H}^{+}$ions and electrons along a midlatitude field line. In the next section we briefly review relevant work, and point out the advances and limitations of simulations performed to date of geophysical plasma densities and flows in the plasmasphere. The third section describes the approximations which we make to adapt our basic equations to each altitude regime and how we formulate the boundary relations between regions so that they are physically and mathematically consistent. The fourth section details the numerical procedure we use to find a unique solution to the system of differential equations and boundary conditions. We present typical results in the fifth section and show that our solution is stable.

\section{Basic Equations}

The specification of magnetic flux tube coordinates in a realistic model of the Earth's field, as shown in Fig. 1, and the computation of local atmospheric parameters from a semi-empirical model $[5,6]$ ensure that the plasma physics 
simulation will give geophysically consistent results. Schunk [7] has presented a general system of transport equations for the Earth's upper atmosphere and ionosphere. This system of equations contains a continuity, momentum, internal energy, stress tensor and heat flow equation for each species in the gas mixture. Application of this system to the midlatitude ionosphere and plasmasphere permits several simplifying assumptions to be made which significantly reduces the complexity of the equations.

We consider a plasma comprised of only two major ions, $\mathrm{O}^{+}$and $\mathrm{H}^{+}$, and electrons in a neutral atmosphere of $\mathrm{O}, \mathrm{O}_{2}, \mathrm{~N}_{2}$ and $\mathrm{H}$. We assume that species temperature and flow velocity differences are small, a midlatitude condition. Thus we can neglect stress and nonlinear acceleration terms, and use Burgers' [8] linear collision terms (cf. St. Maurice and Schunk [9]). In addition, density and temperature gradients perpendicular to the geomagnetic field lines are neglected. To arrive at the above formulation we use the equations derived by St. Maurice and Schunk [9], but we also include terms that take account of collisions with neutrals.

With the above assumptions we derive the following set of matrix equations for continuity and momentum:

$$
\begin{aligned}
\frac{\partial}{\partial t}\left[\begin{array}{l}
N_{1} \\
N_{2}
\end{array}\right] & =\left[\begin{array}{c}
P_{1} \\
0
\end{array}\right]-\hat{L} \cdot\left[\begin{array}{l}
N_{1} \\
N_{2}
\end{array}\right]-\nabla \cdot\left[\begin{array}{l}
\phi_{1} \\
\phi_{2}
\end{array}\right], \\
{\left[\begin{array}{l}
\phi_{1} \\
\phi_{2}
\end{array}\right] } & =-\left[\begin{array}{cc}
N_{1} & 0 \\
0 & N_{2}
\end{array}\right] \frac{\left[\begin{array}{cc}
\left(1+v_{2 x} / v_{21}\right) & 1 \\
1 & \left(1+v_{1 x} / v_{12}\right)
\end{array}\right]}{\left(\frac{v_{1 x}}{v_{12}}+\frac{v_{2 x}}{v_{21}}+\frac{v_{1 x}}{v_{12}} \frac{v_{2 x}}{v_{21}}\right)}
\end{aligned}
$$

where the diffusive force is

$$
\begin{aligned}
Q_{i} & =\frac{\nabla\left(N_{i} T_{i}\right)}{N_{i} T_{i}}-\frac{m_{i} g_{\|}}{k T_{i}}+Z_{i} \frac{T_{e}}{T_{i}} \frac{\nabla\left(N_{e} T_{e}\right)}{N_{e} T_{e}} \\
& \mp \gamma_{i} \frac{\nabla T_{e}}{T_{i}} \pm \frac{N_{j}}{N_{e}}\left(\frac{\alpha_{i j}}{T_{i}} \nabla T_{i}-\frac{\alpha_{i j}^{*}}{T_{i}} \nabla T_{j}\right)
\end{aligned}
$$

and the ordinary $\left(D_{i}\right)$ and thermal $\left(\alpha_{i j}, \alpha_{i j}^{*}, \gamma_{i}\right)$ diffusion coefficients are, as given in St. Maurice and Schunk $[9 \mid$,

$$
\begin{aligned}
D_{i} & =\frac{k T_{i}}{m_{i} v_{i j}}\left(\frac{1}{1-\Delta_{i j}}\right), \\
\gamma_{i} & =\mp \frac{15\left[\left(N_{e} / N_{i}\right) v_{e i}-Z_{i}\left(v_{e i}+v_{e j}\right)\right]}{13\left(v_{e i}+v_{e j}\right)+8 v_{e e}}, \\
\alpha_{i j} & =\frac{15}{8} \frac{N_{e}}{N_{j}} \frac{\mu_{i j}}{M_{i}} \frac{T_{i}}{T_{i j}} \frac{\mu_{i j}\left(v_{j}^{\prime}-v_{j i}^{\prime}\right)}{\left(v_{i}^{\prime} v_{j}^{\prime}-v_{i j}^{\prime} j_{j i}^{\prime}\right)}, \\
\alpha_{i j}^{*} & =\left(\frac{M_{i}}{M_{j}}\right)^{2}\left(\frac{T_{j}}{T_{i}}\right) \frac{\left(v_{i}^{\prime}-v_{i j}^{\prime}\right)}{\left(v_{J}^{\prime}-v_{j i}^{\prime}\right)} \alpha_{i j},
\end{aligned}
$$


as is the correction term $\Delta_{i j}$ :

$$
\Delta_{i j}=\frac{3}{5} \frac{N_{j}}{N_{e}} \frac{\mu_{i j}}{M_{j}}\left[\alpha_{i j}+\frac{N_{i} k T_{i}}{N_{i} k T_{i}} \alpha_{i j}^{*}\right] .
$$

The upper sign and $i=1$ apply to $\mathrm{O}^{+}$, while the lower sign and $i=2$ apply to $\mathrm{H}^{+}$in these equations. The symbol $i=x$ applies to ambient neutrals. $N_{i}, \phi_{i}, Z_{i}, M_{i}$ and $T_{i}$ are the number density, flux, charge, mass and temperature of the $i$ th species. $g_{11}$ is the parallel component of gravity, $\mu_{i j}$ is the reduced mass of species $i$ and $j$, and $T_{i j}=$ $\left(M_{j} T_{i}+M_{i} T_{j}\right) /\left(M_{i}+M_{j}\right)$ is the average temperature of the two species. The collision frequencies, $v_{i j}$, and effective collision frequencies, $v_{i}^{\prime}$ and $v_{i j}^{\prime}$, are also taken from St. Maurice and Schunk [9]. Also the symbol $\hat{L}$ represents a matrix of reaction frequencies determined from the various neutral densities and ion-neutral binary rate constants.

The accurate calculation of a number of locally determined parameters on the basis of realistic geophysical models is required as a prerequisite to the realistic simulation of plasma transport. The Earth's rotating tilted dipole, and gravitational field, are calculated locally and neutral atmospheric parameters may be taken from a semi-empirical model. We selected the MSIS model atmosphere by Hedin et al. [5,6] because it reproduces the seasonal solar cyclic and diurnal variations of the atmosphere as well as its response to geomagnetic disturbances. The attenuated solar flux and photoionization rate are then calculated locally.

\section{Previous Studies}

In this study we restrict our treatment to the plasmasphere, which is a region of closed field lines. Therefore, we need not concern ourselves with supersonic flows [10-12], as they occur primarily outside the plasmasphere.

The modern hydrodynamic or diffusive equilibrium approach to the upper regions of trapped plasma tubes was formulated in 1967 by Walker [13], who pointed out the important role of thermal diffusion in the ionosphere, and in 1969, Schunk and Walker [14] reformulated the general coupled equations of diffusive equilibrium to include any number of major ions, of any charge, as well as thermal diffusion. When collisional terms are included, the diffusive equilibrium equations of Schunk and Walker [14] become the coupled ion momentum equations of Schunk [7]. With the continuity equations, these form the basic tools for treating both the high and low regions.

As mentioned above, previous investigations have dealt mainly with limited portions of magnetospheric flux tubes. At $F$ region altitudes, for instance, it is clear that ion-ion and ion-neutral collisions are both important. The ion fluxes can be calculated accurately from the ion momentum equations (2a) at these altitudes and then substituted into the continuity equations (1) to form coupled parabolic equations in $\mathrm{O}^{+}$and $\mathrm{H}^{+}$. In earlier work, Schunk and Walker $[15]$ and Ruster $[16]$ formulated 
parabolic diffusion equations for $\mathrm{O}^{+}$. In other works by Schunk and Walker [17], Roble [18] and Stubbe [19] coupled parabolic equations were formulated for $\mathrm{O}_{2}^{+}$, $\mathrm{NO}^{+}, \mathrm{O}^{+}$and $\mathrm{H}^{+}$for the $\mathrm{F}$ region and topside ionosphere, but $\mathrm{H}^{+}$was treated as a minor ion in each of these cases, or the $\mathrm{H}^{+}$density was calculated by assuming chemital equilibrium with $\mathrm{O}^{+}$. Such formulations all apply to altitudes below about $2000 \mathrm{~km}$, and require artificially designated or measured upper boundary conditions.

Some early attempts were made to extend this treatment for $\mathrm{O}^{+}$and $\mathrm{H}^{+}$along the entire flux tube $[20,21]$, but this leads to serious numerical problems, which are discussed in the next section. We show in the next section that a satisfactory solution to the entire field tube problem can be achieved by linking appropriate low- and highaltitude formulations with continuity conditions on flux and density at about $1500 \mathrm{~km}$.

The searching method has also been used in attempts to treat the entire flux tube by a single method. In the searching method [22-26] the flux or velocity from the continuity equation is substituted into the collision terms of the momentum equation. The resulting integrodifferential equation is then integrated numerically down from the equator, where flux and density boundary conditions are imposed. The equatorial boundary conditions are readjusted and the integration performed repeatedly until low-altitude chemical boundary conditions are satisfied. The main disadvantages of this approach stem from the fact that flux determinations from the continuity equation are inherently inaccurate and unstable at low altitudes, and that the method does not produce simultaneous solutions in both hemispheres. Therefore, convergence may be up to an order of magnitude slower than that discussed in this paper.

In spite of the basic difficulties with the shooting method, considerable progress has been made in geophysical modeling. Bailey et al. [25] have shown that $\mathrm{H}^{+}-\mathrm{O}^{+}$ counterstreaming can occur with $\left[\mathrm{O}^{+}\right]$flowing upwards above $500 \mathrm{~km}$ during a diurnal simulation. Additional calculations by Bailey et al. [27] have shown that an $L=3$ flux tube will probably never be able to replenish itself between magnetic storms. Their method has some slight variations from a purely shooting solution, in that the $\mathrm{O}^{+}$density was computed using a parabolic diffusion equation below $1500 \mathrm{~km}$ and by diffusive equilibrium above. The $\mathrm{H}^{+}$simulation, however, was achieved using the shooting method. Recent shooting method results by Moffett and Murphy [22] included energy, continuity and momentum equations, and were used to study, among other things, a collapsing postsunset ionosphere.

The perturbation solution of Mayr et al. [28] also treats the entire field line as a single region. Mayr et al. integrate numerically but with only a gradually increasing fraction of the collisional terms in the momentum equations, along the entire field line. The drag terms are always evaluated in the $n$th approximation to obtain the $n+1$ st approximation. This method also suffers from low-altitude problems for the same reasons mentioned in connection with searching methods. It is apparent that both of these methods are basically high-altitude formulations and that neither should be used unless an accurate lower boundary at, say, $1000 \mathrm{~km}$ can be supplied by some other type of calculation or by measurement.

Multiple-region formulations somewhat similar to our own have been used by Park 
and Banks [29] and Marubashi and Grebowski [30], who treated the topside region above $3000 \mathrm{~km}$ as a reservoir. The former did not treat diffusion of $\mathrm{O}^{+}$between 500 and $3000 \mathrm{~km} \mathrm{[29],} \mathrm{however,} \mathrm{and} \mathrm{the} \mathrm{latter} \mathrm{ignored} \mathrm{significant} \mathrm{terms} \mathrm{in} \mathrm{the} \mathrm{diffusive}$ equilibrium formulation at high altitudes [30].

\section{REGIONS AND BOUNDARIES}

We have split each plasma flux tube into three regions, $B, C$, and $B^{*}$, as shown in Fig. 1. The best approximation to use in each region, and the boundary altitude between them, will depend upon both ion and neutral densities. The continuity and momentum equations may be formulated either as parabolic partial differential equations or as integrodifferential equations, i.e., the shooting method. These two methods use different techniques to obtain the ion flux. In the shooting method the ion flux is obtained from the continuity equation, and in the parabolic case from the momentum equations. Although these two procedures are mathematically equivalent, their numerical accuracy varies as a function of altitude.

When the shooting method is used at low altitudes, the ion flux is only the small difference of the much larger integrated photochemical terms. A small error in ion densities causes a large error in the chemical reaction frequencies

$$
\hat{L} \cdot\left[\begin{array}{l}
N_{1} \\
N_{2}
\end{array}\right]
$$

and a much larger error in the flux gradient and the tlux.

When the flux is determined from the momentum equations, as in the parabolic equation method, the accuracy suffers when the diffusive force, $Q_{i}$ of Eq. (2a), tends to zero. This occurs at great altitudes where the diffusive force and diffusion coefficient, $D_{i}$ of Eq. (2a), become very small and very large, respectively, although the ion fluxes remain finite. The individual terms of $Q_{i}$ do not become small at high altitudes. Therefore, $Q_{i}$ becomes the small algebraic sum of several relatively larger terms. The fractional error of $Q_{i}$ becomes large at high altitudes and so does the fractional error of ion flux, which is a linear combination of $Q_{i}$ terms when calculated from the momentum equations. Since one of the major terms of each $Q_{i}$ is $\nabla N_{i} / N_{i}$ it is clear that the flux calculation by this method will be hypersensitive at high altitudes to small errors in the densities.

For a given step size the shooting method wil perform more accurately at high altitude but the parabolic diffusion equations are a better choice for low altitude. Fortunately, there is a considerable overlap from about 500 to $2500 \mathrm{~km}$, where either formulation will perform adequately. We therefore choose a boundary altitude, dictated by atmospheric conditions, somewhere within the limits of the overlap region, and use parabolic differential equations below and a modified form of the 
chooson, and use parabolic differential equations below and a modified form of the searching method above. The transport time for $\mathrm{H}^{+}$is less than $1 \mathrm{hr}[30]$ at the boundary height we have chosen, while the chemical lifetime is about $10^{4} \mathrm{sec}$. With increasing altitude the chemical lifetime tends to become still longer, while the diffusive lifetime becomes shorter. The locations of the interregional boundaries in each hemisphere are indicated by 2 and $2 *$ in Fig. 1 .

The actual procedure by which the boundary altitude was chosen was experimental. We gradually increased the boundary altitude, noting the decreasing response of the high-altitude portion of the density curves above $1000 \mathrm{~km}$ or so. At greater boundary altitudes, $3000 \mathrm{~km}$ or greater, the convergence of the low-altitude portion became slower and somewhat unstable. We chose $2000 \mathrm{~km}$ as the best compromise for stability, accuracy and speed of convergence.

In our high-altitude region, $\mathrm{C}$, the relative flow terms become insignificant for $\mathrm{H}^{+}$ and $\mathrm{O}^{+}$in the plasmasphere, and the diffusive equilibrium approximation is used. Since the diffusion coefficients $D_{i}$ of Eqs. (2) become very large in this region while the actual fluxes are known to remain finite, the diffusive force terms, $Q_{i}$, must be very small as discussed above. The equations of diffusive equilibrium are given in our formulation by

$$
Q_{i}=0
$$

We integrate these first order coupled equations numerically from boundary 2 through region $\mathrm{C}$ to $2^{*}$. This procedure is similar to that of the searching method except that we start at the lower boundary rather than the equator, and we completely ignore the relative flow terms. At a later date we intend to include these terms so that the formulation will correspond to the integrodifferential equations of Bailey et al. $[25]$.

We have adopted the equations of "diffusive equilibrium" although we have done so on the basis of somewhat nonstandard assumptions. Usually all fluxes and time derivatives are assumed at the outset to be zero so that all acceleration and relative flow terms of the momentum equations can be omitted. By contrast we have used the equally sufficient assumptions of subsonic flow velocities, reasonable for the entire plasmasphere, and very low densities applicable to region $\mathrm{C}$, in order to ignore exactly the same terms. The density profiles calculated in $\mathrm{C}$ have the shape of diffusive equilibrium profiles at any instant, but they match the time variable densities at boundary 2. Therefore non-zcro fluxcs arc required to inflate of deflate these variable profiles.

The full equations of continuity are also valid in region $\mathrm{C}$. Therefore we have a total of four first order differential equations in the four independent variables $N_{1}$, $N_{2}, \phi_{1}$ and $\phi_{2}$ in region $\mathrm{C}$, where $N_{1}, N_{2}$ are the densities, and $\phi_{1}, \phi_{2}$ the corresponding fluxes.

In regions $B$ ans $\mathrm{B}^{*}$, all collisions are important, so the full momentum equations (2a) provide an accurate computation of flux. When these equations are substituted 
into Eqs. (1), the equations of continuity, we obtain two coupled parabolic density equations.

$$
\begin{aligned}
& \frac{\partial}{\partial t}\left[\begin{array}{l}
N_{1} \\
N_{2}
\end{array}\right]=\left[\begin{array}{c}
P_{1} \\
0
\end{array}\right]-\hat{L} \cdot\left[\begin{array}{l}
N_{1} \\
N_{2}
\end{array}\right] \\
& +\nabla \cdot\left[\begin{array}{cc}
N_{1} & 0 \\
0 & N_{2}
\end{array}\right]\left[\begin{array}{cc}
\left(1+v_{2 x} / v_{21}\right) & 1 \\
1 & \left(1+v_{1 x} / v_{12}\right)
\end{array}\right]\left[\begin{array}{c}
D_{1} Q_{1} \\
D_{2} Q_{2}
\end{array}\right] \text {, } \\
& \left(\frac{v_{1 x}}{v_{12}}+\frac{v_{2 x}}{v_{21}}+\frac{v_{1 x}}{v_{12}} \begin{array}{c}
v_{2 x} \\
v_{21}
\end{array}\right)
\end{aligned}
$$

where second order terms will originate in the spatial derivatives involved in $Q_{1}$ and $Q_{2}$.

In regions $A$ and $A^{*}$, which include points 1 and $1^{*}$, respectively, the diffusive lifetime greatly exceeds the chemical lifetime; hence, we are justified in dropping the flux divergence terms from the continuity equations which then become local photochemical equations.

$$
\frac{\partial}{\partial t}\left[\begin{array}{l}
N_{1} \\
N_{2}
\end{array}\right]=\left[\begin{array}{c}
P_{1} \\
0
\end{array}\right]-\hat{L} \cdot\left[\begin{array}{l}
N_{1} \\
N_{2}
\end{array}\right]
$$

These equations require an initial condition, but no additional boundary condition or specified parameter, for the regions $A$ and $A^{*}$ to which they apply.

Such is not the case in the adjacent $B$ and $B^{*}$ regions. The parabolic equations of each region require the specification of two independent parameters per variable such as boundary densities or density gradients, per region, in order to determine a unique mathematical solution. Similarly the four first order equations of region $C$ require the specification of one independent parameter for each of the four variables $N_{1}, N_{2}, \phi_{1}$ and $\phi_{2}$, to determine a unique mathematical solution in that region.

Any physically reasonable solution must be continuous in flux $\left(\phi_{1}\right.$ and $\left.\phi_{2}\right)$ and density $\left(N_{1}\right.$ and $\left.N_{2}\right)$ at the boundaries 2 and $2^{*}$. The flux in region $\mathrm{C}$ just above the boundary can of course by expressed in terms of density gradients just below the boundary by means of the momentum equation (2a), since flux is not an independent variable in regions $B$ and $B^{*}$. The continuity conditions at boundary 2 provide four independent relations between the four free parameters of region $B$ and the four free parameters of region $C$. The continuity conditions at $2 *$ provide similar relations between the parameters of regions $C$ and $B^{*}$.

At boundaries 1 and $1^{*}$ we may specify the density by the local photochemical equations as previously elucidated. This provides the remaining two independent parameters for regions $B$ and $B^{*}$. In summary we have used 12 independent continuity relations to reduce three independent solutions with four free parameters 
each to a single unique solution which spans all three regions $B$ and $B^{*}$ and is continuous in flux and density at all points.

When the momentum equations, which are ordinary first order equations, are integrated from boundary 2 , it is clear that densities at all points of $\mathrm{C}$, including $2^{*}$, are determined by the densities at point 2 alone. This may be expressed in functional form as:

$$
N_{i}(S)=G_{i}\left(N_{1}(2), N_{2}(2), S\right) .
$$

In the computer code this function is obtained by numerical integration of Eqs. (3), whose terms are defined in Eq. (2b). When the function is substituted into Eqs. (1) and they are in turn integrated we obtain an equation that shows the relationship of the fluxes to the inflation of the entire region $\mathrm{C}$.

$$
\begin{aligned}
\frac{1}{B\left(2^{*}\right)} & {\left[\begin{array}{l}
\phi_{1}\left(2^{*}\right) \\
\phi_{2}\left(2^{*}\right)
\end{array}\right]-\frac{1}{B(2)}\left[\begin{array}{l}
\phi_{1}(2) \\
\phi_{2}(2)
\end{array}\right] } \\
= & \int_{2}^{2^{*}}\left\{\left[\begin{array}{c}
\mathrm{P}_{1} \\
0
\end{array}\right]-\hat{L} \cdot\left[\begin{array}{c}
N_{1}(S) \\
N_{2}(S)
\end{array}\right]-\frac{\partial}{\partial t}\left[\begin{array}{c}
N(S) \\
N_{2}(S)
\end{array}\right]\right\} \frac{d s}{B(S)} .
\end{aligned}
$$

The $1 / B(S)$ factors all arise from the divergences along the nonuniform flux tube whose area varies as $1 / B(S)$. It is to be noted that if $\phi_{i}(2), \phi_{i}\left(2^{*}\right)$ and $N_{i}(2)$ have some specific values then $\partial N_{i}(2) / \partial t$, which controls the last term in the integral of Eq. (7) via dynamic equilibrium, must have a particular unique value to be consistent with Eq. (6).

\section{Numerical ANALYsis}

Equations (2a), (4) and (7) form the basis of our finite difference formulation when the following substitution is made for any spatial derivative:

$$
\left.\frac{\partial f}{\partial s}\right|_{k+1 / 2}=\theta_{f} \frac{\left[f\left(S_{k+1}, t_{l+1}\right)-f\left(S_{k}, t_{l+1}\right)\right]}{S_{k+1}-S_{k}}+\left(1-\theta_{f}\right) \frac{\left[f\left(S_{k+1}, t_{l}\right)-f\left(S_{k}, t_{l}\right)\right]}{S_{k+1}-S_{k}},
$$

where $k$ indexes arc length, $l$ indexes time, and $\theta_{f}$ is a variable parameter input that determines the implicit fraction during execution. The values $\theta_{f}=0, \frac{1}{2}, 1$ are input for explicit, Crank-Nicholson, and Laesonen differencing, respectively. In this section of the paper Eqs. (7), (4) and (2a) will refer to the finite difference version with Eq. (8) substituted for spatial derivatives in each case. The nonlinear algebraic equations which are obtained are solved by the Newton-Raphson method.

We have actually integrated the continuity equation in regions B and $\mathrm{B}^{*}$, as well as $\mathrm{C}$, before using the finite difference approximation. Thus Eq. (4) becomes the following function for the iteration: 


$$
\begin{aligned}
& F=\int_{S_{k}-1 / 2}^{S_{k}+1 / 2}\left\{\left[\begin{array}{c}
P_{1} \\
0
\end{array}\right]-\hat{L} \cdot\left[\begin{array}{l}
N_{1} \\
N_{2}
\end{array}\right]-\frac{\partial}{\partial t}\left[\begin{array}{c}
N_{1} \\
N_{2}
\end{array}\right]\right\} \frac{d s}{B(S)} \\
& +\left.\frac{1}{B(S)}\left[\begin{array}{cc}
N_{1} & 0 \\
0 & N_{2}
\end{array}\right]\left[\begin{array}{cc}
\left(1+v_{2 x} / v_{21}\right) & 1 \\
1 & \left(1+v_{1 x} / v_{12}\right)
\end{array}\right]\left[\begin{array}{c}
D_{1} Q_{1} \\
D_{2} Q_{2}
\end{array}\right]\right|_{s_{k-1 / 2}} ^{s_{k+1 / 2}}, \\
& \left(\frac{v_{1 x}}{v_{12}}+\frac{v_{2 x}}{v_{21}}+\frac{v_{1 x}}{v_{12}} \frac{v_{2 x}}{v_{21}}\right)
\end{aligned}
$$

i.e., a solution is obtained when $F \approx 0$.

Therefore, our finite difference procedure might aptly be called a "flux preserving finite difference code." The form of $\mathrm{Eq}$. (9) now strongly resembles that of (7); the primary difference is the much longer spatial interval in Eq. (7) and the use of dynamic equilibrium to obtain the densities within the interval. We will see that this similarity lends itself well to the unified iterative approximation to the solution in all three regions.

Figure 2 is a schematic of how our spatial grid points are related to the boundaries of our low- and high-altitude formulations. The first and last points of our spatial grid are located at the lower boundaries 1 and $1^{*}$, respectively, while the interregional boundaries 2 and $2^{*}$ each exactly bisect the arc length between a pair of points. Thus the points $2 \mathrm{~A}$ and $2 \mathrm{~B}$ are located just above and just below boundary 2 , and the

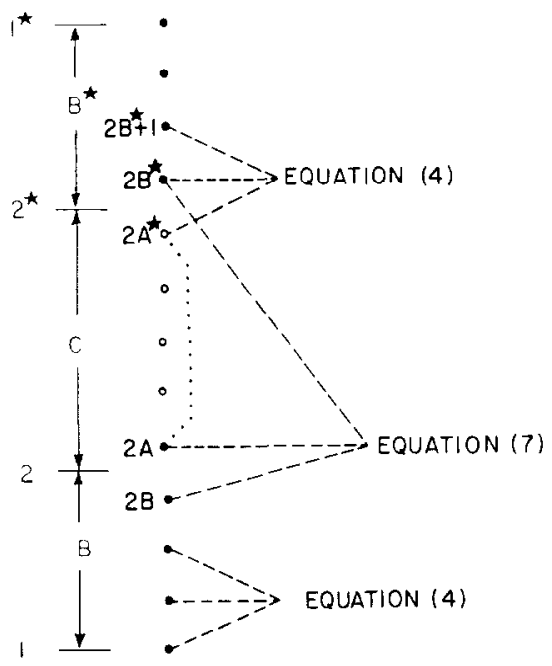

Fig. 2. Schematic of spatial grid. It is to be noticed that boundaries 2 and $2^{*}$ are each straddled by the pairs of points $(2 \mathrm{~A}, 2 \mathrm{~B})$ and $\left(2 \mathrm{~A}^{*}, 2 \mathrm{~B}^{*}\right)$, respectively, from which the boundary fluxes are calculated. Boundaries 1 and $1^{*}$, on the other hand, fall exactly upon the first and last point. The densities at these points are calculated via local photochemical equilibrium. Both densities at each of three points are needed to calculate each finite difference equation used in the Newton-Raphson iterative solution. The three points are adjacent for Eq. (4) but not for Eq. (7). The three points needed to calculate Eq. (7) are $2 \mathrm{~B}, 2 \mathrm{~A}$ and $2 \mathrm{~B}^{*}$. 
points $2 \mathrm{~A}^{*}$ and $2 \mathrm{~B}^{*}$ are located just above and just below boundary $2^{*}$, respectively. All the densities of region $\mathrm{C}$, including $2 \mathrm{~A}^{*}$ are determined via dynamic equilibrium from the densities at the single point $2 \mathrm{~A}$. The densities at the four points $2 \mathrm{~A}, 2 \mathrm{~B}, 2 \mathrm{~A}$ * and $2 B^{*}$, substituted in Eq. ( $2 a$ ), are sufficient for determining both boundary fluxes in Eq. (7). Integral terms in (7), based on dynamic equilibrium profiles, are also keyed to the densities at $2 \mathrm{~A}$, so actually three points suffice for Eq. (7).

The densities at three sequential points inside B or B*, as shown in Fig. 2, suffice to determine the parabolic equations (4). Also shown are the three non-sequential points, $2 \mathrm{~B}, 2 \mathrm{~A}$ and $2 \mathrm{~B}^{*}$, whose densities suffice to determine Eq. (7), as explained above, and the non-sequential triple $2 \mathrm{~A}, 2 \mathrm{~B}^{*}$ and $2 \mathrm{~B}^{*}+1$, which serves to determine the type (4) equation spanning boundary $2^{*}$.

The Jacobian matrix for our Newton-Raphson iteration is computed numerically by taking small variations of the densities involved in each of the equations. It is to be noted that only the point $2 \mathrm{~A}$ of region $\mathrm{C}$ receives an independent density variation while the densities of the rest are regenerated by dynamic equilibrium. Therefore only the densities at that one point, $2 \Lambda$, of the region $C$ appear in the array, between the points of regions B and $\mathrm{B}^{*}$. Two equations of type (7) appear in the equation array, and form just a double row for $\mathrm{O}^{+}$and $\mathrm{H}^{+}$at that point, in the Jacobian matrix.

After convergence of the iterative solution, the equations of dynamic equilibrium are used one more time to compute the density profiles for the rest of region $\mathrm{C}$. They key element in the iterative scheme is Eq. (7). Although its integral terms are based on the first order equations of dynamic equilibrium, they respond to variations in the density in the same way as the corresponding terms of the parabolic equations. The differences of the two boundary fluxes also respond in the same way to variations in density, as do the analogous flux gradient terms of the parabolic equations.

\section{RESULTS}

We first discuss the tests we performed to demonstrate that the simulation converges to a stable steady state solution. We then analyze the steady state ion fluxes, and finally we present simulations of the collapse of the sunset ionosphere.

For this stability test, the rotation of the earth and the time evolution of all locally computed parameters were frozen at UT 17:33 Aug. 10, and furthermore set artificially to N-S symmetry about the magnetic equator of an $L=2$ field line at approximately $70 \mathrm{~W}$ longitude. Our temperature model for this test featured $T_{i}=4500 \mathrm{~K}, T_{e}=5500 \mathrm{~K}$ at the magnetic equator and $T_{i}=T_{e}=500 \mathrm{~K}$ at $120 \mathrm{~km}$. To obtain a steady state solution the time derivative terms of Eqs. (4) and (7) were set equal to zero and the simulation allowed to iterate to convergence.

Using the steady state solution as an initial condition we then allowed the simulation to run in the time dependent mode, but with all the local ambient parameters still frozen at UT 17:33. Using many 5-min time steps we found that the time dependent density profile simulation did not drift and displayed an oscillation of less than one part in $1,000,000$ about the steady state solution. The profiles also 
proved to be symmetric to better than one part in $1,000,000$. We feel that the results of these tests constitutee a rigorous test of stability and conservation of particles.

The steady state profiles show some interesting aspects of ion production and flow which indicate that the code is reproducing expected geophysical variations. The steady state flux profiles, denoted $\mathrm{Oh}$ in Fig. 3, show downflow of both $\mathrm{O}^{+}$and $\mathrm{H}^{+}$ below $500 \mathrm{~km}$ but counterstreaming above with $\mathrm{O}^{+}$moving upwards and $\mathrm{H}^{+}$ downwards. The peak $\mathrm{O}^{+}$downflux occurs very strongly near the altitude of the $\mathrm{O}^{+}$ density peak, about $250 \mathrm{~km}$ as shown in Fig. 4. This can be attributed to the fact that the chemical loss rate, due to reaction of $\mathrm{O}^{+}$with $\mathrm{N}_{2}$ and $\mathrm{O}_{2}$, increases with decreasing altitude more rapidly than the photoionization production rate as one approaches the $\mathrm{O}^{+}$density peak from above. The net result is that the $\mathrm{O}^{+}$ions are actually flowing into their region of most rapid production.

Above about $500 \mathrm{~km}$, the calculated $\mathrm{O}^{+}$flux is upward, as is typically seen in daytime measurements $|31|$, and the $\mathrm{H}^{+}$is counterstreaming downward. At altitudes above $550 \mathrm{~km}$, the counterstreaming $\mathrm{H}^{+}$and $\mathrm{O}^{+}$flux are virtually equal in

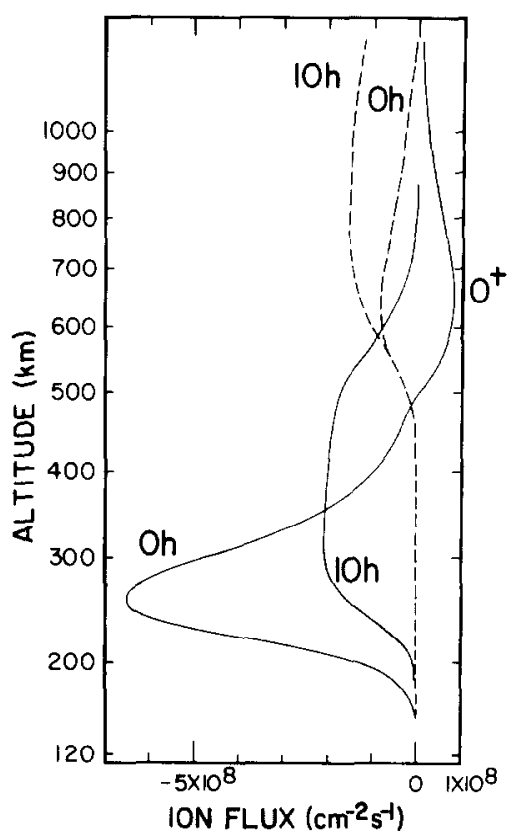

Fig. 3. Simulated ion fluxes. Both $\mathrm{H}^{+}$and $\mathrm{O}^{+}$fluxes are shown, $\mathrm{O} \mathrm{hr}$ denoting steady state conditions, and $10 \mathrm{hr}$ the ion fluxes $10 \mathrm{hr}$ after a simulated ionospheric collapse. For steady state conditions, denoted $0 \mathrm{hr}$ in the figure, the $\mathrm{H}^{+}$flux is downward everywhere while the $\mathrm{O}^{+}$flux is divided into a downward regime below $500 \mathrm{~km}$ and an upward regime above. It is apparent that above about $600 \mathrm{~km}$, where $\mathrm{O}^{+}+\mathrm{H} \leftrightarrow \mathrm{H}^{+}+\mathrm{O}$ are the only chemical reactions, the two fluxes are virtually equal and opposite. Another striking feature, compared with Fig. 4 is that the maximum $\mathrm{O}^{+}$flux occurs right at the maximum in the $\mathrm{O}^{+}$density profile. Ten hours after collapse, denoted $10 \mathrm{hr}$, both fluxes are everywhere downward. The $\mathrm{O}^{+}$downflux is higher than previously at high altitudes. but lower below $350 \mathrm{~km}$ because of a decrease in ion density. 


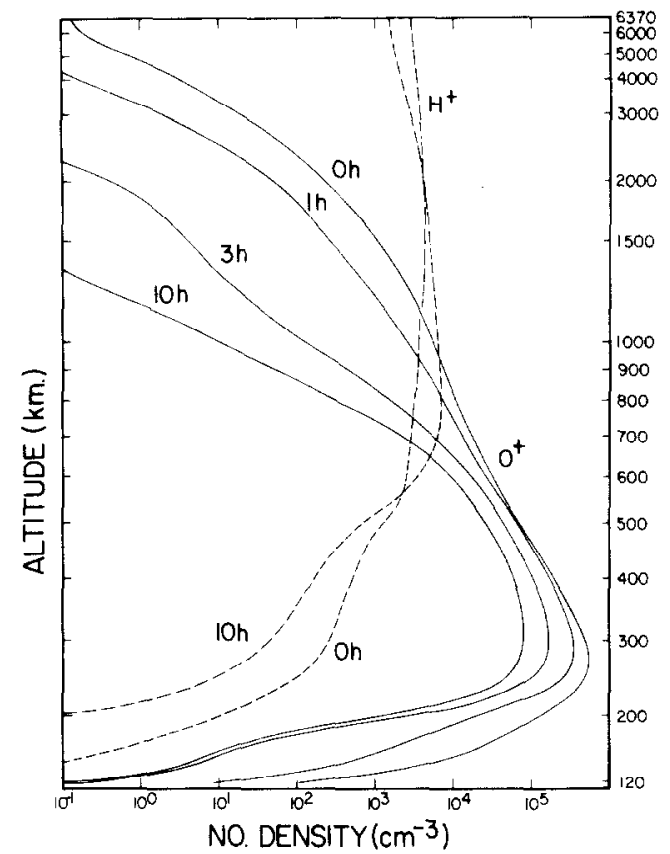

Fio. 4. Simulated ion densities. For $\mathrm{H}^{+}$, curves for steady state conditions and $10 \mathrm{hr}$ after the collapse, denoted $0 \mathrm{hr}$ and $10 \mathrm{hr}$ respectively, are shown. For $\mathrm{O}^{+}$the curves denoted $0 \mathrm{hr}, 1 \mathrm{hr}, 3 \mathrm{hr}$ and $10 \mathrm{hr}$ denote steady state and then 1,3 , and $10 \mathrm{hr}$ after the simulated ionospheric collapse.

magnitude except for a small residual difference caused by the small amount of photoproduction even at very high altitudes.

Photoproduction and electron recombination are very slight above $550 \mathrm{~km}$. Under steady state conditions $\partial N_{i} / \partial t=0$ as well, so with symmetric conditions, $\phi_{\mathrm{H}}+(s)+\phi_{\mathrm{O}^{+}}(s)=0$ by conservation of charge. Quite simply, counterstreaming is required by the continuity equations, the particular direction of the counterstreaming being determined by the relative densities of neutral $\mathrm{H}$ and neutral $\mathrm{O}$ at higher and lower altitudes. The counterstreaming also produces a slight inflection in the $\mathrm{H}^{+}$ steady state curve at about $500 \mathrm{~km}$, where the slope of the $\mathrm{H}^{+}$density profile increases suddenly. This we attribute to the upflow of the major ion, $\mathrm{O}^{+}$, just above the $500 \mathrm{~km}$ level and downflow just below, which tends to force the $\mathrm{H}^{+}$along with it.

We have also simulated an ionospheric collapse, as might occur at sunset. For this purpose we allowed the electron heating rate and the photoproduction rate to decay exponentially at each point, with a time constant of $20 \mathrm{~min}$. The results are shown in Fig. 4 as curves annotated with the time after the initiation of collapse. Times of 0,1 , 3 , and $10 \mathrm{hr}$ are shown for $\mathrm{O}^{+}$, while only 0 and $10 \mathrm{hr}$ are shown, for clarity of display, in the case of $\mathrm{H}^{+}$.

The collapse of the topside $\mathrm{O}^{+}$density appears to be the most profound sunset effect, while the topside $\mathrm{H}^{+}$density profile only appears to settle slightly. Both 
effects, however, are due to the same cause: the reduction of the topside ion and electron temperatures. In our temperature model, the equatorial temperatures are both reduced to about $1100 \mathrm{~K}$ after $10 \mathrm{hr}$ from initial values of $T_{i}=4500 \mathrm{~K}$ and $T_{e}=5500 \mathrm{~K}$ used for the steady state. The concomitant reduction in topside scale heights causes the $\mathrm{O}^{+}$density, which had a shorter scale height to begin with, to decrease more rapidly.

It will be noticed that the reduction of densities is moderate at middle altitudes and then again very pronounced near the lower boundary of our simulation. Temperature effects have less influence here. The more dramatic decrease at the lower altitudes is to be expected, however, because the chemical destruction rate is much greater at the lower altitudes than at the middle altitudes.

The counterstreaming reported for steady state conditions disappears, to be replaced within about $1 \mathrm{hr}$ by downstreaming of both ions everywhere. Even after $10 \mathrm{hr}$ however, there remains a residual pattern that looks as if the steady state counterstreaming were superimposed upon a net downflux. It would appear that the profiles are still in the process of adjusting to the reduced temperatures. The reduced photoproduction can no longer support an upflux of $\mathrm{O}^{+}$at high altitudes in any case.

The $\mathrm{H}^{+}-\mathrm{O}^{+}$transition height is also lowered at night. Where it was $1150 \mathrm{~km}$ under daytime steady state conditions it has dropped to only $650 \mathrm{~km}$ after $10 \mathrm{hr}$. The lower transition height is a combined effect resulting from the reduction of both the neutral and ion scale heights under the lower-temperature postcollapse conditions. Note that the $\mathrm{H}^{+}$density actually increases at around $800 \mathrm{~km}$. The basic features agree with Evans and Holt's geophysical observations $|31|$ and demonstrate clearly that the code is working properly.

\section{Future ImProvements in the Simulation}

Our simulated density profiles show that our approximation of dynamic equilibrium is satisfactory for $\mathrm{H}^{+}$and $\mathrm{O}^{+}$above the altitude where $\mathrm{H}^{+}$becomes the only major ion. Nevertheless there is an almost imperceptible discontinuity in the density gradients at boundaries 2 and $2^{*}$, so we intend to include ion-ion collisions at the very high altitudes at a later date. Two possible ways to do this present themselves. One is to formulate $\mathrm{O}^{+}$density as a parbolic equation at all altitudes (regions $\mathrm{B}, \mathrm{C}$ and $\mathrm{B}^{*}$ ), but retain first order momentum and continuity equations for $\mathrm{H}^{+}$at high altitudes (region $C$ ). Another way is to upgrade the dynamic equilibrium solutions iteratively by calculating each $V_{i}$ from the continuity equation integrated between boundary 2 and $2 *$, and then to substitute it into the momentum equation

$$
\begin{aligned}
V_{i}(S) & =\frac{B(S)}{N_{i}(S)}\left\{\frac{\phi_{i}(2)}{B(2)}+\int_{2}^{s}\left[P_{i}-\hat{L}_{i j} N_{j}-\frac{\partial N_{i}}{\partial t}\right] \frac{d s}{B(S)}\right\}, \\
Q_{i} & =-\frac{1}{D_{i}}\left[\left(V_{i}-V_{j}\right)+\frac{v_{i x}}{v_{i j}} V_{i}\right] .
\end{aligned}
$$


The velocity $V_{i}$ in Eq. (10) would be calculated from the previous iteration. Note that $Q_{i}$ will now be equal to some small correction number, rather than identically equal to zero, as in Eq. (3).

The code will be used to study a number of geophysical problems including diurnal variations under a number of geophysical conditions, effects of interhemispheric flows, magnetospheric substorms and seasonal and solar cyclic variations upon profiles flux tube content and filling processes. Future simulations will be compared extensively with satellite and ground-based measurements. The electron and ion energy equations will be simulated simultaneously as well. Future runs should therefore shed considerable light upon diurnal variations, plasmaspheric heat sources, ion fluxes and the maintenance of the nighttime ionosphere.

\section{CONCLUSIONS}

We have achieved the most comprehensive simulation to date of field-aligned plasma transport in the plasmasphere. The plasma simulation itself incorporates the best aspects of two older methods in a unified mathematical model and uses accurate geophysical parameters to produce an accurate and meaningful solution to the geophysical plasma problem.

We have shown that we can link the solutions for an entire flux tube connecting the ionosphere and protonosphere even though it passes through several regions that require different sets of differential equations to approximate conditions in different regimes. We have derived the continuity relations across the boundary regions between different approximations which are necessary for this type of approach and replace artificially specified boundary conditions used in previous work. We have shown that a high-altitude counterstreaming of $\mathrm{H}^{+}$and $\mathrm{O}^{+}$must and does result under steady state conditions.

\section{ACKNOWLEDGMENTS}

The authors gratefully acknowledge helpful discussions with A. F. Nagy, R. W. Schunk, J. P. St. Maurice and A. E. Hedin. This work was supported by AF Contract F19628-77-C-0007. Computing support was provided by the National Center for Atmospheric Research, which is sponsored by the National Science Foundation.

\section{REFERENCES}

1. D. L. Carpenter and C. G. Park, Rev. Geophys. Space Phys. 11 (1973), 133.

2. C. R. Chappel, Rev. Geophys. Space Phys. 10 (1972), 951.

3. P. M. Banks, in "Critical Problems of Magnetospheric Physics," Proceedings of the Joint COSPAR/IAGA/URSI Symposium, 1972.

4. P. M. Banks, R. W. Schunk, And W. J. Raitt, "The Topside Ionosphere: A Region of Dynamic Transition," Annual Review of Earth and Planetary Sciences, Vol. 4, 1976. 
5. A. E. Hedin, H. E. Salah, J. V. Evans, C. A. Reber, G. P. Newton, N. W. Spencer, D. C. Kayser, D. Alcayde, P. Bauer, L. Cogger, and J. P. McClure, J. Geophys. Res. 82 (1977), 2139.

6. A. E. Hedin, C. A. Reber, G. P. Newton, N. W. Spencer, H. C. Brinton, H. G. Mayr, and W. E. POTTER, J. Geophys. Res. 82 (1977), 2148.

7. R. W. Schunk, Planet. Space Sci. 23 (1975), 437.

8. J. M. Burgers, "Flow Equations for Composite Gases," Academic Press, New York, 1969.

9. J. P. St. Maurice and R. W. Schunk, Planet. Space Sci. 25 (1977), 907.

10. W. J. Raitt, R. W. Schunk, ANd P. M. Banks, Planet. Space Sci. 23 (1975), 1103.

11. P. M. Banks, A. F. Nagy, and W. I. Axford, Planet. Space Sci. 19 (971), 1053.

12. E. G. Fontheim and P. M. Banks, Planet. Space Sci. 20 (1972), 73.

13. J. C. G. Walker, Planet. Space Sci. 15 (1967), 1151.

14. R. W. Schunk and J. C. G. Walker, Planet. Space Sci. 17 (1969), 853.

15. R. W. Schunk and J. C. G. Walker, Planet. Space Sci. 17 (1970), 535.

16. R. Rüster, J. Atmos. Terr. Phys. 33 (1971), 137.

17. R. W. Schunk and J. C. G. Walker, Planet. Space Sci. 21 (1973), 187.

18. R. G. Roble, Planet. Space Sci. 23 (1975), 1017.

19. P. Stubbe, J. Atmos. Terr. Phys. 32 (1970), 865.

20. P. Bauer, "The Cooling of the Protonosphere," Ph. D. thesis, University of Michigan, 1968.

21. J. MAssa, "Theoretical and Experimental Studies of the Ionization Exchange between the lonosphere and Plasmasphere," Ph.D. thesis, University of Michigan, 1974.

22. R. J. Moffett and J. A. Murphy, Planet. Space Sci. 21 (1973), 43.

23. H. G. Mayr, L. H. Brace, and G. S. Dunham, J. Geophys. Res. 72 (1967), 4391.

24. J. A. Murphy AND R. J. Moffett, Planet. Space Sci. 26 (1978), 281.

25. G. J. Balley, R. J. Moffett, And J. A. Murphy, Planet. Space Sci. 25 (1977), 967.

26. P. Richardo, Ph.D, thesis, Melbourne, 1978.

27. G. J. Bailye, R. H. Moffett, And J. A. Murphy, Planet. Space Sci. 26 (1978), 753.

28. H. G. Mayr, E. G. Fontheim, L. H. Brace, H. C. Brinton, and H. A. Taylor, Jr., J. Aimos. Terr. Phys. 34 (1972), 1659.

29. C. G. Park and P. M. Banks, J. Geophys. Res. 79 (1974), 4661.

30. K. Marubashi and J. M. Grebowski, J. Geophy's. Res. 81 (1976), 1700.

31. J. V. Evans and J. M. Holt, Planet. Space Sci. 26 (1978), 727. 